\title{
"YO SABÍA QUE NADIE ME IBA A JUZGAR»: LA ADICCIÓN AL JUEGO ONLINE DESDE LA PERSPECTIVA DE GÉNERO ${ }^{1}$
}

“I Knew Nobody Would Judge Me": Addiction to Online Gambling from a Gender Perspective

\author{
Alicia Rius-Buitrago ${ }^{1}$, Isabel Soriano-Villarroel² ${ }^{2}$ Hibai López-González $^{3}$ \\ Autora correspondencia: Alicia Rius-Buitrago \\ Correo electrónico: alicia@imcasociacion.org
}

1. Instituto Mujeres y Cooperación. (Madrid, España)

2. Fundación de Educación para la Salud (FUNDADEPS). (Madrid, España)

3. Institut d’investigació Biomèdica de Bellvitge (IDIBELL). (Barcelona, España)

Recibido: 15/04/2020 Aceptado: 31/07/2020

\footnotetext{
${ }^{1}$ El trabajo que ha dado lugar a este artículo ha recibido una financiación de $12000 €$ del Plan Nacional sobre Drogas (Ministerio de Sanidad) a UNAD (Unión de Asociaciones y Entidades de Atención al Drogodependiente).
} 


\section{RESUMEN}

OBJETIVOS. Aproximarse a los problemas específicos de las mujeres con trastorno de juego online desde la perspectiva de género. MATERIAL Y MÉTODO. Estudio cualitativo realizado en 2019 mediante 10 grupos de discusión formados por pacientes en tratamiento y profesionales. Seis grupos fueron con pacientes mayores de edad en tratamiento por juego online. Se complementaron con una entrevista individual a una mujer en tratamiento. Cuatro fueron a profesionales (psicólogos/as y trabajadores/as sociales) con experiencia en el tratamiento del juego online. En total, se analizaron los discursos de 41 pacientes ( 3 mujeres y 38 hombres, con una edad media de 30 años) y 28 profesionales (20 mujeres y 8 hombres, con una media de 36 años). RESULTADOS. Se presentan los resultados de los 12 nodos codificados en el conjunto de la investigación relativos a cuestiones de género, resumidos en 5: Masculinidad hegemónica; Jugadoras online: escasa presencia y visibilidad; Doble penalización, estigma y violencia; Mayor comorbilidad asociada a los mandatos de género: jugar para evadirse; Mujeres solas y dificultades para acceder a los recursos. CONCLUSIONES. Durante el estudio se han confirmado hallazgos previos de la literatura que asimilaban la adicción a sustancias con el juego patológico online en las mujeres. Esta similitud se basa, entre otros, en los motivos que desencadenan la adicción, la mayor comorbilidad, el estigma y doble penalización, las intersecciones con las violencias de género, la soledad a la hora de afrontar los tratamientos y la invisibilidad en los servicios.

Palabras clave: Juego online; Género; Masculinidad; Mujer; Tratamiento.

\section{ABSTRACT}

AIM. To approach the specific problems of women with online gambling disorder from a gender perspective. MATERIAL AND METHOD. Qualitative study carried out in 2019 through 10 discussion groups made up of patients under treatment and professionals. Six were with patients of legal age in treatment for online game related disorders. They were complemented by an individual interview with a woman in treatment. Four went to professionals (psychologists and social workers) with experience in dealing with online gambling. In total, the speeches of 41 patients ( 3 women and 38 men, with an average age of 30 years) and 28 professionals (20 women and 8 men, with an average of 36 years) were analyzed. RESULTS. The results of the 12 nodes coded in the whole of the research related to gender issues are presented, summarized in 5 : Hegemonic masculinity; Online players: little presence and visibility; Double penalization, stigma and violence; Greater comorbidity associated with gender mandates: playing to escape; Single women and difficulties in accessing resources. CoNCLUSIONS. During the study, previous findings from the literature that assimilated substance addiction with online pathological gambling in women have been confirmed. This similarity is based, among others, on the reasons that trigger addiction, greater comorbidity, stigma and double penalization, intersections with gender violence, loneliness when facing treatments and invisibility in services.

Keywords: Online Gambling; Gender; Women; Masculinity; Treatment. 


\section{INTRODUCCIÓN}

En España, la memoria anual de la Dirección General de la Ordenación del Juego (DGOJ 2016) recoge que el 0,9\% de las personas adultas (alrededor de medio millón) son jugadoras patológicas, es decir, que cumplen cuatro o más de los síntomas descritos en el DMS-5. Según la encuesta EDADES 2019 se estima que el 0,3 \% del total de la población de entre 15 y 64 años ha padecido este trastorno en el último año.

Según el mismo informe, el número de jugadoras y jugadores activos en el país ha pasado de 637000 en 2013 a 1465000 en 2018, con una media aproximada de dos cuentas online activas por cada persona usuaria registrada, siendo el $85 \%$ hombres y el $15 \%$ mujeres. Este crecimiento es posible debido a los cambios legislativos sucedidos en mayo de 2011, que regularon el juego online y abrieron el mercado para que las empresas del sector solicitasen licencias para operar mediante dominios es dentro del territorio español.

El juego online supera al juego tradicional en la velocidad y frecuencia de juego, lo cual es en sí mismo un factor de riesgo elevado debido a su mayor adictividad (Gray, LaPlante, Shaffer, 2012; Lopez-Gonzalez, Estévez, Griffiths, 2018b). Esto promueve que la generación de deuda sea más rápida y el problema tenga una evolución más corta e intensa, aunque las mujeres gastan de media 276 euros anuales, casi la mitad que los hombres, con 484 euros anuales (DGOJ 2019). Este dato quizás es debido al menor poder adquisitivo de las mujeres (ONU Mujeres, 2019; FMI, 2019; Gómez, 2002). Además, si analizamos el tipo de juego por sexo, los hombres prefieren los de acción (el doble que las mujeres), mientras que ellas eligen los juegos sociales hasta tres veces más que los hombres (Frank N. Magid Associates, 2013), manteniéndose también vigentes en este ámbito los estereotipos de género (Bruel dos Santos, 2008).

Según apuntan varios autores y autoras, alrededor de un $30 \%$ de las personas afectadas por trastorno de juego son mujeres, pero solo un $10 \%$ busca ayuda terapéutica (Kim, 2016; Granero, 2009; Tavares, 2001; Potenza, 2011). ¿Por qué? Desafortunadamente es escasa la investigación relativa al juego online y las mujeres. Por ello es necesario avanzar en el análisis de los motivos que dificultan el acceso de las mujeres a los recursos de atención como la socialización diferencial de género, la invisibilidad del consumo, la diferencia en el apoyo social y familiar recibido (doble penalización en las mujeres) o la dificultad en el acceso y permanencia al tratamiento en 
mujeres (comorbilidad y correlación con otros problemas como violencia de género) (Martínez Redondo, 2008).

Las mujeres con trastorno por juego online patológico, al igual que las que consumen de forma abusiva algún tipo de sustancia, tienen múltiples desventajas a la hora de iniciar, mantener o beneficiarse de los tratamientos existentes, ya que el sesgo en la atención ha tomado como referencia a la población masculina. Unido a esto, las problemáticas biopsicosociales asociadas al problema de adicción que presentan las mujeres están ocultas porque todavía se detecta una falta de formación en perspectiva de género por parte del conjunto de profesionales, elementos que complican el tratamiento (Fundadeps, 2018; Martínez Redondo, 2008).

El objetivo de este artículo es contribuir al conocimiento sobre los problemas de juego de azar online en las mujeres para mejorar su tratamiento y contribuir a su visibilidad.

\section{MATERIAL Y MÉTODO}

Este estudio asume un enfoque cualitativo e incorpora la perspectiva de género de forma trasversal en el planteamiento y el análisis de los resultados de la investigación.

El artículo hace referencia a los resultados obtenidos en materia de género durante la investigación Dificultades en el tratamiento del juego online: recomendaciones de profesionales y personas atendidas (Lopez-Gonzalez et al, 2019).

El enfoque cualitativo se materializó a través de la realización de grupos de discusión (GD) y una entrevista individual con dos colectivos diferentes: (1) personas atendidas por problemas de juego online con un diagnóstico de trastorno de juego y (2) profesionales con experiencia en tratar dicho trastorno. La Tabla 1 muestra los principales descriptores de los grupos de discusión. 
Tabla 1. Diseño de la muestra de la investigación, técnicas aplicadas y contenidos de los guiones de conducción

\begin{tabular}{|c|c|c|c|}
\hline Colectivo & Técnica & $\begin{array}{c}\text { Número de } \\
\text { participantes* }\end{array}$ & $\begin{array}{l}\text { Contenido de los guiones de } \\
\text { conducción }\end{array}$ \\
\hline \multirow[t]{2}{*}{$\begin{array}{l}\text { Personas } \\
\text { atendidas por } \\
\text { trastorno de } \\
\text { juego }\end{array}$} & $\begin{array}{l}6 \text { grupos de } \\
\text { discusión }\end{array}$ & $40(2,38)$ & $\begin{array}{l}\text { - Diferencias entre juego on-line vs off-line } \\
\text { - Relación con otros consumos online } \\
\text { - Atracción de la interfaz } \\
\text { - Depósitos y retiradas } \\
\text { - Combinación de juego on-line y off-line } \\
\text { - Facilitación social en internet } \\
\text { - Barreras percibidas de entrada al } \\
\text { tratamiento }\end{array}$ \\
\hline & $\begin{array}{l}1 \text { entrevista } \\
\text { individual }\end{array}$ & $1(0,1)$ & $\begin{array}{l}\text { - Motivación para jugar } \\
\text { - Atracción al juego } \\
\text { - Hábitos de juego } \\
\text { - Socialización on-line vs off-line } \\
\text { - Barreras de entrada para recibir ayuda } \\
\text { - Apoyo familiar } \\
\text { - Barreras durante el tratamiento }\end{array}$ \\
\hline $\begin{array}{l}\text { Profesionales de } \\
\text { la salud mental }\end{array}$ & $\begin{array}{l}4 \text { grupos de } \\
\text { discusión }\end{array}$ & $28(20,8)$ & $\begin{array}{l}\text { - Diferencias de perfiles respecto a } \\
\text { jugadores/as presenciales } \\
\text { - Barreras de entrada al tratamiento } \\
\text { - Barreras durante el tratamiento. } \\
\text { Cogniciones sobre juegos azar/habilidad } \\
\text { - Diferencias entre mujeres y hombres }\end{array}$ \\
\hline
\end{tabular}

Nota: *Entre paréntesis el número de mujeres y el número de hombres.

Se llevaron a cabo un total de 10 GD en las ciudades de A Coruña, Leganés, Madrid, Oviedo, Sevilla, Toledo y Vigo. Por tanto, en total se contó con 69 personas, 23 mujeres y 46 hombres). Se pretendía realizar entrevistas a mujeres de forma individual con el fin de que tuvieran un espacio seguro y confidencial en el que poder expresar sus vivencias con el juego, y las posibles discriminaciones y violencias asociadas, pero pese a los reiterados intentos por conseguir una muestra con más mujeres jugadoras, debido a su escasa presencia en los recursos de atención, finalmente solo se pudo entrevistar a tres. Una de forma individual y otras dos en el contexto de los GD.

Los GD, diseñados y conducidos por el equipo investigador, tuvieron una duración media de 73 minutos. Todas las transcripciones se analizaron por dos investigadores/as del equipo y posteriormente se pusieron en común y se discutió la interpretación de los resultados. La técnica de análisis utilizada se ha basado en el análisis temático (AT; Braun \& Clarke, 2006). Para ello se ha utilizado el software NVivo 11 para Mac, a través el cual se extrajeron un total de 58 temas preliminares. 
Durante la redacción, se utiliza el masculino genérico para referirse exclusivamente a jugadores hombres; en los casos en los que incluye tanto a hombres como a mujeres, se duplica el sujeto o se utilizan fórmulas que no connotan sexo.

\section{RESULTADOS}

Los resultados del estudio original se agruparon en tres bloques: la opinión de quienes juegan; la opinión de quienes tratan a quienes juegan; y las cuestiones de género. En el presente artículo, se presentan de manera agrupada en cinco nodos los resultados relacionados con este último bloque de género: Masculinidad hegemónica; Jugadoras online: escasa presencia y visibilidad; Doble penalización, estigma y violencia; Mayor comorbilidad asociada a los mandatos de género: jugar para evadirse; Mujeres solas y dificultades para acceder a los recursos.

Debido a la escasa participación de las mujeres con trastorno por juego online en el estudio, los resultados se apoyan principalmente en el análisis del discurso de profesionales y en el contraste de este con la visión de algunas personas en tratamiento. Esto supone una limitación en el análisis de los resultados, ya que carece del discurso de las propias mujeres.

\section{Masculinidad hegemónica}

El hecho de que los hombres estén socializados en valores predominantemente masculinos es un dato que quizás explique y ayude a entender porque la mayoría de las personas que desarrollan conductas de juego patológico son varones. El riesgo y la individualidad forman parte de estos valores. Las actitudes vinculadas a lo masculino suponen un obstáculo propio para los hombres en tanto que:

(a) les entorpece el trabajo personal hacia la recuperación, pues no hacen una reflexión introspectiva: (Paciente 38, Leganés) «Desde que hemos llegado aquí (hablo del tema de juego) no hemos pensado ninguno el tema de los sentimientos, el carácter o la forma que tienes tú de ser. Y muestran conductas más resistentes a la hora asumir las normas»; (Prof. 65, Madrid): «Empiezas por el control económico: qué cantidad asignas, qué no asignas. El hombre siempre demanda más de lo que le pones. "Es que, claro, ¿Cómo voy a ir sin dinero? ¿Cómo no voy a tener libertad? ¿Cómo no voy a tener?”. Y la 
mujer viene un poco como diciendo: "Lo que digáis, lo que digáis, incluso prefiero no manejar nada...”».

(b) tienen diversas resistencias a reconocer el problema: (Paciente 40, Leganés) «Pues yo era la primera vez que bajaba a hablar con mi padre y bajé nervioso, nunca había ido a hablar con él, nunca le había contado nada... Era mi primer momento hablando con él...». Unido a esto, los hombres son conscientes de los permisos que el sistema les concede por el hecho de ser hombres (anonimato, ausencia de juicio social, etc.) elementos externos todos ellos facilitadores del juego online (Paciente 33, Sevilla): «En las maquinas, en el móvil... pero la cuestión principal era esa, yo sabía que nadie me iba a juzgar». Este tipo de actitudes ligadas a la masculinidad tradicional, como fanfarronear con los amigos o seguidores en redes sociales sobre las ganancias como símbolo de poder aportan seguridad y favorecen el mantenimiento de la adicción.

El ejercicio de poder también se ha presentado en los hombres entrevistados en modo de violencia psicológica hacia sus parejas. Algunos jugadores relataban que, cuando sus parejas sospechaban de que estaban jugando, ellos lo negaban rotundamente, acusándolas de estar «locas». Esta estrategia puede encuadrarse dentro de un comportamiento de gaslighting relacionado con violencia psicológica: (Paciente 40, Leganés) «Yo he llegado a estar en el coche con mi novia, y mi novia preguntarme que qué estaba haciendo y yo decirle “¿cómo voy a estar apostando?, ¿te das cuenta de que estás loca?”».

En sus procesos de rehabilitación, esta identificación con la masculinidad tradicional también influye en la resistencia mostrada por los hombres a recibir ayuda. Los hombres jóvenes, a pesar de estar acompañados en los procesos de rehabilitación con mucha más frecuencia que las mujeres, no siempre reciben de buen grado este apoyo y a menudo no reconocen el valor del trabajo de cuidados, respondiendo con conductas agresivas y violentas que ponen en riesgo tanto el proceso como a las personas de las que dependen (en el caso de los menores de edad). (Prof. 48, Toledo): «Nada, se cabrea muchísimo en las sesiones de grupo, muy agresivo, falta de control de impulsos y de autocontrol, y al final, se acaba apartando del grupo, los padres están fatal... y ha intentado por ejemplo pagar la deuda con otros delitos, por ejemplo, traficando con droga, lo que le ha metido en otros problemas claro...».

En esta misma línea se ha detectado que los hombres presentan mayores conductas delictivas que las mujeres, como el robo o la venta de drogas, que, unido a la solicitud de 
microcréditos para poder afrontar las deudas, favorece el rápido endeudamiento y con ello la detección temprana del problema, sobre todo en población menor de edad.

Destaca la escasa importancia que se da, tanto por pacientes como por profesionales, al hecho de que los pacientes desatiendan el trabajo de cuidados familiares. Esto se refleja en la naturalización de que los hombres no tienen responsabilidades familiares más allá de las aportaciones económicas (estereotipo del breadwinner) y en la autoindulgencia con la que muchos hombres han mencionado situaciones de falta de corresponsabilidad en el cuidado. Además, en los discursos de las y los profesionales apenas aparecen relatos relacionados con hombres que no han cuidado de sus familias, que han permanecido ausentes de la crianza de sus hijas e hijos, etc.

A lo largo de la investigación, se han detectado algunos casos en los que se reproducen estereotipos de género por parte de las y los profesionales, por ejemplo, en relación con el mandato social de la maternidad. En el caso que se expone a continuación se estaba hablando sobre las estrategias para explicar la problemática del juego a pacientes jóvenes. (Prof. 54, Toledo): «¿Sabes lo que me funciona a mí, particularmente, muy bien? Los ejemplos de las mujeres jugadoras. Ahí se ve perfectamente que, si no fuese una enfermedad, una madre no actuaría de esa manera y no se olvidaría de recoger a los niños del colegio». En otros casos se ha ponderado especialmente la dejación de los deberes de cuidado de una mujer adicta. (Prof. 54, Toledo): «Tenemos un caso [...]. La madre que tenía una niña con necesidad de una leche especial. Aguaba los biberones. Entonces, cuando ella empieza a rehabilitarse, se da cuenta como madre cómo ha podido hacer eso, que a su hija ella la ha criado con biberones aguados».

\section{Jugadoras online: escasa presencia y visibilidad}

En relación con las mujeres que juegan de forma online, el primer resultado con el que nos encontramos es la escasa presencia y visibilidad que tienen en el entorno de juego presencial. Esto se puede deber a varios factores: las mujeres perciben los salones de apuestas como lugares inhóspitos e incómodos donde no se sienten a gusto. Además, las pocas veces que entran en un salón suele ser acompañando a la pareja (un hombre), y nunca van en grupo de chicas ni participan en los grupos de WhatsApp en los que se discute de apuestas deportivas.

Hay profesionales que consideran que las mujeres optan por el juego online al sentirse incómodas en algunos sitios de juego mayoritariamente masculinos. Existe, de hecho, la 
sensación de que puede haber más mujeres en el juego online de las que se detecta porque guarda similitudes con el «esconder» la adicción que se da en el consumo de sustancias. Jugar online te da anonimato y, en este sentido, podría haber más consumo femenino del que reportan los estudios.

También se ha valorado que, al tratarse el juego online de una práctica más aislada y menos sociable, en las mujeres puede que se den factores de control que no se dan cuando el juego es un factor de socialización. (Prof. 56, Madrid): «Con las mujeres [...] este aspecto quizás más gregario de quedar con mucha gente también era algo más solitario en este sentido».

Respecto al modo de apostar, el papel que juega el autocontrol en las mujeres, derivado de las responsabilidades de cuidado y reproducción social que el sistema de género les asigna, hace que la manera de apostar y jugar difiera de la masculina. La manera de apostar se correlaciona con el nivel de endeudamiento, por lo que la consecuencia lógica es que los hombres se endeudan más y de manera más rápida. Esta opinión se ve avalada por la experiencia en atención de varios profesionales. (Prof. 44, Toledo): «Llevo un año y unos meses en este mundo de la ludopatía y yo sí que veo un perfil de chavales jóvenes, (chicas no...) que tienen una deuda bastante grande en poco tiempo».

\section{Doble penalización, estigma y violencia}

En esta similitud con el consumo de sustancias, parece que las jóvenes con problemas de juego online temen visibilizarse en los servicios, entre otras cosas, por la doble penalización social que supone quebrantar los roles de género al presentar una adicción al juego online. (Prof. 52, 54, 53 Toledo): «Creo que hay menos chicas que chicos jugando online, aunque también las chicas dan menos la cara, lo hacen más a escondidas»; «Es que estamos en lo de siempre, socialmente hay una doble penalización».

Esta doble penalización impacta de forma directa en la autoestima de las mujeres, hecho que no favorece la recuperación, y que requiere de una atención enfocada también a la ruptura de estos mandatos. (Prof. 52, Toledo): «La mujer tiene que ser la que cuide de los niños, la que haga todo... Entonces, la mujer no puede ser la que esté enganchada. Porque no, porque tiene que dedicarse a sus hijos».

Como profesionales, han visto muchas veces cómo se desautoriza a las mujeres que destapan su problema. (Prof. 65, Madrid): «Sí, es que claro, el estatus que pierden cuando 
tienen un problema de adicciones es brutal. O sea, son malas madres, son malas trabajadoras, son malas mujeres, son malas».

También hay una creencia fuertemente arraigada entre profesionales y personas adictas que se ha repetido en varios grupos y que tiene que ver con penalizar a las mujeres porque gastan un dinero que no es suyo. (Prof. 42, Toledo): «La mujer cree que se está gastando el dinero de la familia, el hombre piensa que se está ganando su dinero».

Por otro lado, y al igual que sucede con el consumo de sustancias, en las mujeres con trastorno por juego online se detectan situaciones de violencia de género (previo y/o presente) en el ámbito de la pareja, violencia sexual, abuso sexual en la infancia, o maltrato familiar. (Prof. 62, Madrid): «Se asemeja en otro aspecto que a mí me ha resultado curioso, que es la presencia de malos tratos, muy parecido a adicciones con sustancia».

En los grupos de discusión de profesionales, incluso se habla de que esta prevalencia se ha estudiado a través de alguna pequeña investigación de sus entidades (Prof.64, Madrid): «Sí que hemos tenido algún estudio reducido a nuestro propio ámbito de actuación: el centro de día de la asociación, en el que vemos que el maltrato era una cuestión bastante frecuente, no solo durante la situación de juego, sino incluso anterior, en la propia relación de pareja».

A pesar de que las preguntas sobre la presencia de violencia no están contempladas habitualmente en las fichas de atención, sí se han comentado varios casos en los que este dato ha surgido, bien en la terapia, bien en los grupos (Prof. 59, Madrid):

Yo si me acabo de acordar, de una mujer de juego online, que empezó en casino. Es una mujer de cincuenta y pocos años, separada, se separó ella porque había maltrato psicológico. No fue bien visto ni por su expareja, que todavía... le trae problemas con respecto a sus hijos, ni por sus hermanos, ni por su hermana, que además eran amigas. Entonces se vio muy sola y empezó con el juego.

También se relatan otro tipo de violencias machistas sufridas por parte de algunas mujeres jugadoras de videojuegos online como agresiones verbales y micromachismos. (Prof. 54, Toledo): «¿Y los videojuegos? También hay muchísimas chicas donde además hay un gran problema. Ahí sí que hay un gran problema de género. Son maltratadas según 
cuentan muchas de ellas, digamos que por la terminología o el chaval que juega con ellas: “Tú sabes menos...”, los micromachismos».

\section{Mayor comorbilidad asociada a los mandatos de género: jugar para evadirse}

En relación con la comorbilidad, además de estas violencias, el conjunto de profesionales que han participado en los grupos de discusión relataba que las mujeres acuden con mayores problemáticas asociadas al juego que los hombres, muchas de ellas derivadas de las relaciones de género. (Prof. 52, Toledo): «Las mujeres suelen llegar... Para mí, mucho más depresivas. [...]. Con depresión, falta de atención por parte de su pareja...».

Se relatan muchos casos en los que las mujeres llegan al tratamiento con presencia de otros trastornos previos. (Prof. 60, Madrid): «Una mujer, que primero hizo una adicción a sustancias y luego se enganchó en juego online. [...] Era una mujer como muy enfermiza, muy patológica y con una historia familiar muy dura, y esta sí que fue su compañera la que le dijo, “o dejas esto y vas a terapia o me separo de ti,” y sigue en terapia, y la verdad que bastante bien».

En los hombres también se dan situaciones de depresión o ansiedad, pero se detectan más tarde que en las mujeres por la dificultad, como se ha visto, de introspección que algunos de ellos presentan (Prof. 48, Toledo): «¿Más comorbilidad en mujeres que hombres? En el hombre también hay mucha comorbilidad, pero se dan cuenta después, cuando ven cómo se ha desestructurado su vida desde del juego, entonces a lo mejor dicen “¡ah pues a lo mejor sí que tenía ansiedad,” “a lo mejor sí que tenía depresión,” y se dan cuenta ya en esta época... La mujer es consciente antes».

La comorbilidad asociada en las mujeres muestra que, en muchas ocasiones, la motivación inicial hacia el juego online sea evadirse de sus problemas. Las y los profesionales entrevistados señalan diferencias de motivación al juego detectadas en mujeres y hombres, concluyendo que los hombres persiguen el dinero fácil mientras las mujeres buscan distracción.

\section{Mujeres solas y dificultades para acceder a los recursos}

Como sucede con el juego presencial, las mujeres de juego online que acuden a tratamiento reciben menos apoyo familiar que los hombres, lo que supone un hándicap para su recuperación. Las que sí reciben apoyo tienden a acudir con otras mujeres 
(hermana, madre, hija, amiga). Algunos hombres, cuando las acompañan, no muestran deseo de implicarse en la terapia, sino que cumplen la función de dejarlas y recogerlas (Prof. 54, Toledo): ““Yo a esto no hace falta que venga, ¿no?” Y me quedé así y digo: “Juan ¿Y si te pasa a ti, tiene que venir Mari?” Y dice: “Pues tendría que venir.” Pues entonces algo pasa. Y no ha vuelto por allí».

En relación con la calidad del acompañamiento, también se mencionan las diferencias. (Prof. 65, Madrid): «Si las mujeres vienen a tratamiento el apoyo no es igual. No sé cómo decirte, cuando la que apoya es una mujer hay más seguimiento. O sea, cuando es el marido viene y "Bueno, yo voy, te acompaño, pero ya está. No me creo que tenga que hacer otra cosa.” Y luego lo notas en las pautas, vamos, en el seguimiento. O sea, cuando la pareja es hombre no se implica tanto en las pautas».

Por el contrario, los relatos de hombres que se han encontrado con el apoyo de sus mujeres y demás familia son mayoritarios en los grupos. (Paciente 40, Leganés): «[... y y me encontré que mi madre, mi hermano, todo el mundo me estaba dando su apoyo, lo que necesitase, las horas que necesitase, si hacía falta que viniese a terapia vendrían conmigo y eso, pues quieras o no quieras, te hace coger más fuerzas».

La relación de ayuda y acompañamiento que las mujeres proporcionan está presente tanto en mujeres como en hombres adictos. Por el contrario, el apoyo que ellas reciben en situaciones de juego patológico repite el mismo patrón que con el consumo de sustancias. (Prof. 52, Toledo): «Yo, una mujer que tuvimos, el marido, de hecho, la acompañó al principio y le dijo: "Pero yo tengo que decir una cosa muy clara, en el momento que vuelva a liarla, yo desaparezco.” Y, efectivamente, así fue. Recayó y el hombre desapareció. Cuando hemos visto... Yo he visto a gente que ha recaído hasta tres veces y la mujer sigue». Esto probablemente explique porque las mujeres en muchas ocasiones acuden a la ayuda profesional de manera clandestina.

En uno de los grupos de profesionales se habló del caso de las mujeres en este sentido. (Prof. 65, Madrid): «Hablamos siempre del acceso de las mujeres a este tratamiento. O sea, si ya el estigma está en “yo aquí no me identifico,” una mujer no se identifica en ningún sitio. Es como: “¿Cómo voy a reconocer que tengo este problema?” Y, claro, llegan como intentando hacer muchas cosas antes por su cuenta para que nadie se entere».

Puesto que los recursos están altamente masculinizados, el tratamiento grupal con las mujeres se vuelve complicado. Esto dificulta la llegada y permanencia de mujeres (Prof. 
53, Toledo): «Toda mujer que llega allí, automáticamente, ve los usuarios que hay allí y huyen. No tenemos mujeres terapeutas y luego todos los usuarios son hombres...». Eso en el caso de que acudan, porque ya se ha comentado la dificultad de captar a mujeres en los recursos: «En nuestra entidad no huyen, no huyen porque no llegan...».

Sumado a esta masculinización de los espacios, e incluso de profesionales, se ha hablado también del juicio emitido a las mujeres en las terapias grupales. Esta conducta tutelar sobre las mujeres, considerándolas inferiores o como si fueran menores de edad, aunque tengan la misma edad y situación que los hombres, está vinculada también a la masculinidad tradicional. (Prof.52, Toledo): «A mí me ha pasado de una que venía, que de hecho era por bingo online; y realmente dejó de asistir porque se sentía atacada por los hombres... en este caso la atacaban».

\section{DISCUSIÓN}

En este estudio se detectaron conductas de juego tanto en hombres como en mujeres que confirman los marcos teóricos previos sobre la similitud entre adicciones con sustancia y trastorno de juego. En general, podemos afirmar que hay una mayor incidencia de hombres que de mujeres afectados por trastorno de juego patológico como demuestran algunos estudios realizados a población general (Husky et al., 2015).

En cuanto a los estilos de juego, los hombres muestran preferencia por los juegos que permiten la socialización presencial, como las casas de apuestas deportivas, los casinos y otros lugares de juego. Las mujeres en cambio buscan más el anonimato y pasar desapercibidas, al igual que sucede con el consumo de sustancias.

Por otra parte, existe una diferencia en el tipo de juegos online a los que se apuesta en función del sexo. Las mujeres parece que juegan de forma virtual a los mismos juegos que de forma presencial. También juegan al póker, ruleta, apuestas deportivas, etc., pero en menor medida que los hombres. En cambio, juegan más al bingo y al parchís (Marcos et al., 2019), dato que se refuerza con la percepción de los hombres jugadores que han participado en el estudio. Estas diferencias por sexo en los tipos de juego no han desembocado en tratamientos específicos para unas y otros (Raylu y Oei, 2002; Echeburúa, 2005). 
Por otra parte, la prudencia y la conservación asociadas a lo femenino frente al riesgo y el peligro asociados a lo masculino hacen que las mujeres se endeuden menos (SENDA, 2016; Bergara, 2018). Las mujeres acuden menos a pedir microcréditos.

En cuanto al apoyo recibido, podríamos decir que el sistema de género que otorga a las mujeres el rol de cuidadoras se ve alterado cuando son estas quienes requieren ayuda por cuestiones que podrían considerarse «masculinas». La mayor reprobación social unida a la falta de apoyo hace que a las mujeres les resulte más difícil reconocer el problema y que acudan en menor medida a pedir ayuda o lo hagan de manera clandestina. Puede que esto explique, al menos en parte, que las mujeres lleguen en mucha menor proporción que los hombres a los recursos de atención al juego patológico que lo que les correspondería por prevalencia (Becoña, 1998; Echeburúa, 2005). Se calcula que alrededor del 70 \% de las mujeres afectadas por juego patológico no llegan a ningún recurso.

La menor presencia de mujeres desemboca en una masculinización de los recursos, factor que explica la dificultad de adherencia y permanencia en el tratamiento, ya que las mujeres que sí llegan se encuentran muchas veces en terapias con mayoría de hombres, sintiéndose incómodas o poco identificadas y no regresando. En este sentido se necesita más investigación para tener certeza en cuanto a la magnitud del problema en las mujeres, sus motivaciones para jugar, sus hábitos de juego, etc., y confirmar que su ausencia en los recursos se debe a un problema estructural de accesibilidad como sucede en el consumo de drogas, o a otro tipo de barreras específicas del juego online.

La mayor presencia de comorbilidad en mujeres que en hombres se ha visto respaldada en la investigación por los grupos de profesionales. Las mujeres adictas al juego presentan también cuadros de ansiedad y depresión, a veces anteriores a su adicción (Marcos et al., 2019).

La motivación hacia el juego online tiene un carácter más evasivo que de búsqueda de sensaciones en las mujeres (Becoña, 1998). En muchas ocasiones, tiene que ver con un refugio ante situaciones familiares difíciles. Un porcentaje elevado de mujeres con juego patológico son o han sido víctimas de violencia de género (Sirvent, 2000; Vázquez, 2012; Martínez, 2008). Pese a ello, no existen protocolos de detección y atención a la violencia de género a pesar de su alta prevalencia (Sirvent, 2000; Hakansson, 2016). 


\section{CONCLUSIONES}

El estudio pone de manifiesto la importancia del análisis de género para comprender y abordar los problemas de las mujeres y los hombres en el juego online patológico. Los resultados refuerzan la idea de que la masculinidad tradicional o hegemónica es un factor de riesgo para el desarrollo de conductas adictivas que emerge como un obstáculo a la hora de reconocer el problema y plantear el trabajo personal hacia la recuperación. Este trabajo ha complementado el conocimiento disponible en la literatura científica sobre la discriminación y el estigma asociados a las mujeres jugadoras, expandiendo sus implicaciones hacia el ámbito del juego online. El estudio sugiere que la reprobación social hacia las mujeres jugadoras, acompañada por el incumplimiento del rol de género femenino que dichas mujeres parecen transgredir con su conducta de juego, y en algunos casos, agravada por la presencia de violencia doméstica hacia ellas, hacen necesaria la incorporación de una perspectiva de género en el tratamiento del juego online.

\section{AGRADECIMIENTOS}

Queremos mostrar nuestro agradecimiento a la Federación Española de Jugadores de Azar Rehabilitados (FEJAR), que ha facilitado el contacto con sus pacientes en tratamiento y ha cedido locales y materiales para la reunión de los grupos de discusión. Asimismo, queremos dar las gracias a las entidades asociadas a la UNAD por el acceso a sus profesionales. Por último, agradecer a todas y todos los pacientes que han contribuido a la investigación aportando sus historias vitales.

\section{BIBLIOGRAFÍA}

Arostegui, E.; MARTínez, P. Mujeres y drogas: Manual para la prevención de recaídas con perspectiva de género. Bilbao: Universidad de Deusto, 2018.

Becoña Iglesias, E. Características de la mujer jugadora patológica. En: Revista de Psicopatología y psicología clínica. 1997, vol. 2, núm. 1, pp. 21-34.

Bergara Sautua, A. Masculinidades y adicciones: ¿La droga nos hace más hombres? En: Proyecto Hombre: revista de la Asociación Proyecto Hombre. 2018, vol. 96, pp. 18-20.

Braun, V.; Clarke, V. Using Thematic Analysis in Psychology. En: Qualitative Research in Psychology. 2006, vol. 3, pp. 77-101. 
BRIME, B., et al. Informe sobre adicciones comportamentales. Juego y uso compulsivo de internet en las encuestas de drogas y adicciones en España EDADES y ESTUDES. 2019. Disponible en:

$<$ www.pnsd.mscbs.gob.es/profesionales/sistemasInformacion/sistemaInformacion/p df/2019_Informe_adicciones_comportamentales.pdf $>$.

BRUEL dos SAnTOS, T. C. Representaciones sociales de género: Un estudio psicosocial acerca de lo masculino y lo femenino. Madrid: Universidad Autónoma de Madrid, 2008. [Tesis doctoral]. Disponible en:

<repositorio.uam.es/bitstream/handle/10486/3965/27615_bruel_dos_santos_teresa_ cristina.pdf?sequence $=1>$.

DiRECCIÓN GENERAL DE ORDENACión DEL JUEGo (DGOJ). Análisis del perfil del jugador online. Madrid: Ministerio de Hacienda, 2018. Disponible en:

$<$ www.ordenacionjuego.es/es/informe-jugador-online $>$.

Dirección General de ORdenación Del Juego (DGOJ). Datos del mercado del juego online estatal. Madrid: Ministerio de Hacienda, 2019. Disponible en:

$<$ www.ordenacionjuego.es/es/mercado-juego-online-estatal $>$.

ECHEBURÚA, E. Retos de futuro en el tratamiento del juego patológico. En: Adicciones. 2005, vol. 17, núm. 1, pp. 11-15.

ECHEBURÚA, E., et al. Clinical gender differences among adult pathological gamblers seeking treatment. En: Journal of Gambling Studies. 2011, vol. 27, núm. 2, pp. 215227.

EDGREN, R., et al. Gender comparison of online and land-based gamblers from a nationally representative sample: Does gambling online pose elevated risk? En: Computers in Human Behavior. 2017, vol. 72, pp. 46-56.

Frank N. Magid Associates. Video Game Business Models and Emerging trends among consumers. 2013. Disponible en:

<www.businesswire.com/news/home/20130321005299/en/PlaySpan-Magid-

Gamer-Survey-Finds-100-Million>.

Fondo Monetario InTERnACIONAL. Las Mujeres y el crecimiento económico. En: Finanzas y Desarrollo. 2019. Disponible en:

<www.imf.org/external/pubs/ft/fandd/spa/2019/03/pdf/fd0319s.pdf>.

FUNDACIÓN DE EDUCACIÓN PARA LA SALUD (FUNDADEPS). Guía breve para la detección e intervención con mujeres en situación de drogodependencias. 2018. Disponible en: < fundadeps.org/wp-content/uploads/eps_media/recursos/documentos/897/GuiaFedra-2018.pdf $>$.

GómEz GómEz, E. Género, equidad y acceso a los servicios de salud: una aproximación empírica. En: Revista Panamericana de Salud Pública. 2002, vol. 11, pp. 327-334.

GRANERO, R., et al. Sex differences among treatment-seeking adult pathologic gamblers. En: Comprehensive Psychiatry. 2009, vol. 50, núm. 2, pp. 173-180. 
Hakansson, A. Diferencias de género en el Trastorno de Juego: Desde los aspectos clínicos hasta la respuesta al tratamiento. En: Cuadernos de medicina psicosomática y psiquiatría de enlace. 2016, núm. 117, pp. 31-39

HuSKY, M. M., et al. Gender differences in the associations of gambling activities and suicidal behaviors with problem gambling in a nationally representative French sample. En: Addictive Behaviors. 2015, vol. 45, pp. 45-50.

KIM, H. S., et al. Gender differences among helpline callers: Prospective study of gambling and psychosocial outcomes. En: Journal of Gambling Studies. 2016, vol. 32, núm. 2, pp 605-623.

LóPEz-GonzÁlez, H.; EstÉvez, A.; GrifFiths, M. D. Marketing and advertising online sports betting: A problem gambling perspective. Journal of Sport \& Social Issues. 2017, vol. 41, núm. 3, pp. 256-272.

LóPez-GonzÁlez, H.; Rius, A.; SoRiAno, I. Dificultades en el tratamiento del juego online: recomendaciones de profesionales y pacientes. Madrid: Unión de Asociaciones y Entidades de Atención al Drogodependiente (UNAD), 2019.

Marcos Serrano, M.; Chóliz Montañés, M. Mujer y juego online. Propuesta de tratamiento de un caso de adicción a videobingo. En: Informació psicològica. 2019, vol. 117, pp. 99-104.

Martínez Redondo, P. Perspectiva de género aplicada a las drogodependencias. Salamanca: Asociación de Entidades de Centros de Día de Drogodependencias, 2008. Disponible en:

<www.generoydrogodependencias.org/wpcontent/uploads/2010/07/perspectiva-deg\%c3\%89nero-aplicada-a-las-drogodependencias.pdf>.

MoYA, M.; ExPÓSITO, F.; PADILLA, J. Revisión de las propiedades psicométricas de las versiones larga y reducida de la Escala sobre Ideología de Género International. En: Journal of Clinical and Health Psychology. 2006, vol. 6, pp. 709-727.

OBSERVATORIO VASCO DE DROGODEPENDENCIAS. Estudio documental sobre drogas y violencia de género. Vitoria-Gasteiz: Gobierno vasco, 2007. Disponible en: $<$ www.drogasgenero.info/wp-content/uploads/drogas-y-violencia-de-genero.pdf $>$.

ORganización de las NACIONES UnidAs (ONU). Mujeres, Familias en un mundo cambiante: El progreso de las mujeres en el mundo 2019-2020. Disponible en: $<$ www.unwomen.org/es/digital-library/progress-of-the-worlds-women>.

PotenzA, M., et al. Gender-related differences in the characteristics of problem gamblers using a gambling helpline. En: American Journal of Psychiatry. 2001, vol. 158, núm. 9, pp. 1500-1505.

Raylu, N.; OeI, T. Pathological gambling. A comprehensive review. En: Clinical Psychology Review. 2002, vol. 22, pp. 1009-1061.

SERVICIO NACIONAL PARA LA PREVENCIÓN Y REHABILITACIÓN DEL CONSUMO DE DROGAS Y Alcohol (SENDA). Hombres con Consumo Problemático de Drogas. 
Tratamiento con Perspectiva de Género. Santiago: Gobierno de Chile, 2016. Disponible en: <http://copolad.eu/es/publicacion/1323>.

SIRVENT, C. Las dependencias relacionales (D. R.): Dependencia emocional, codependencia, bidependencia. En: I Simposium nacional sobre adicción y Mujer. Madrid: Instituto de la Mujer/Fundación Instituto Spiral, 2000. Disponible en: $<$ http://fispiral.com.es/wp-content/uploads/2002/03/I-symposium-sobre-adiccionen-la-mujer.pdf>.

TAVARES, H., et al. Gender differences in gambling progression. En: Journal of Gambling Studies. 2001, vol. 17, núm. 2, pp. 151-159.

VÁzQUEZ, M. Ludopatía y mujer: un análisis desde la perspectiva de género. IV Congreso Universitario Nacional Investigación y Género. Sevilla: Universidad de Sevilla, 2012, pp. 2097-2107 